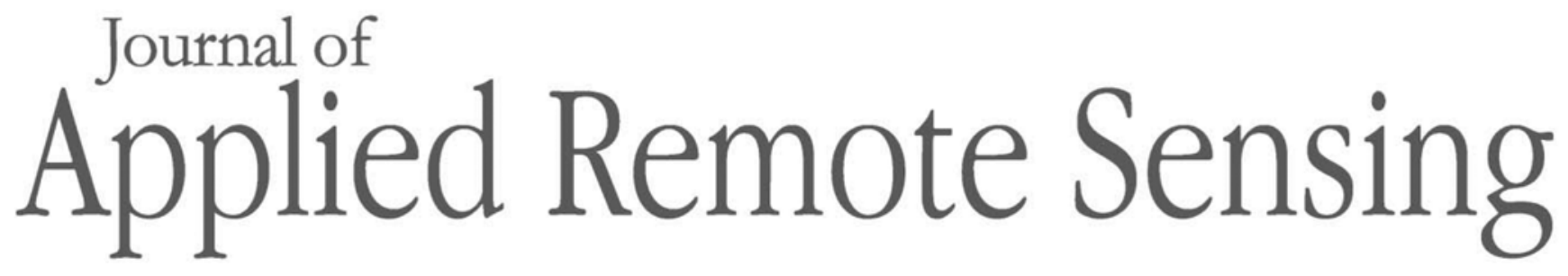

RemoteSensing.SPIEDigitalLibrary.org

\title{
Errata: Integrating pan-sharpening and classifier ensemble techniques to map an invasive plant (Spartina alterniflora) in an estuarine wetland using Landsat 8 imagery
}

\author{
Jinquan Ai \\ Wei Gao \\ Zhiqiang Gao \\ Runhe Shi \\ Chao Zhang \\ Chaoshun Liu
}




\title{
Errata: Integrating pan-sharpening and classifier ensemble techniques to map an invasive plant (Spartina alterniflora) in an estuarine wetland using Landsat 8 imagery
}

\author{
Jinquan Ai, ${ }^{\text {a,b }}$ Wei Gao, ${ }^{\text {a,b,c }}$ Zhiqiang Gao, ${ }^{\text {d }}$ Runhe Shi, ${ }^{\text {a,b }}$ Chao Zhang, ${ }^{\text {a,b }}$ \\ and Chaoshun Liu ${ }^{\text {a,b }}$
}

${ }^{a}$ East China Normal University, College of Geographical Sciences, Shanghai 200241, China

${ }^{b}$ East China Normal University, Key Laboratory of Geographic Information Science,

Ministry of Education, Shanghai 200241, China

${ }^{c}$ Colorado State University, Natural Resource Ecology Laboratory, Fort Collins, Colorado 80523, United States

${ }^{\mathrm{d}}$ Yantai Institute of Coastal Zone Research, Chinese Academy of Sciences, Yantai 264003, China

[DOI: $10.1117 / 1 . J R S .10 .029901]$

This article [J. Appl. Remote Sens. 10(2), 026001 (April 07, 2016)] contained two errors in Fig. 1, in which the vector map of China had an obvious deformation on the northwest and did not display the islands in the South China Sea. Also, support information "Shanghai Municipal Commission of Science and Technology (15DZ1207805) and Health and Family Planning (15GWZK0201)" was added to the Acknowledgements section. All online versions of the article were corrected on 19 April 2016.

The correct form of Fig 1. is shown below:

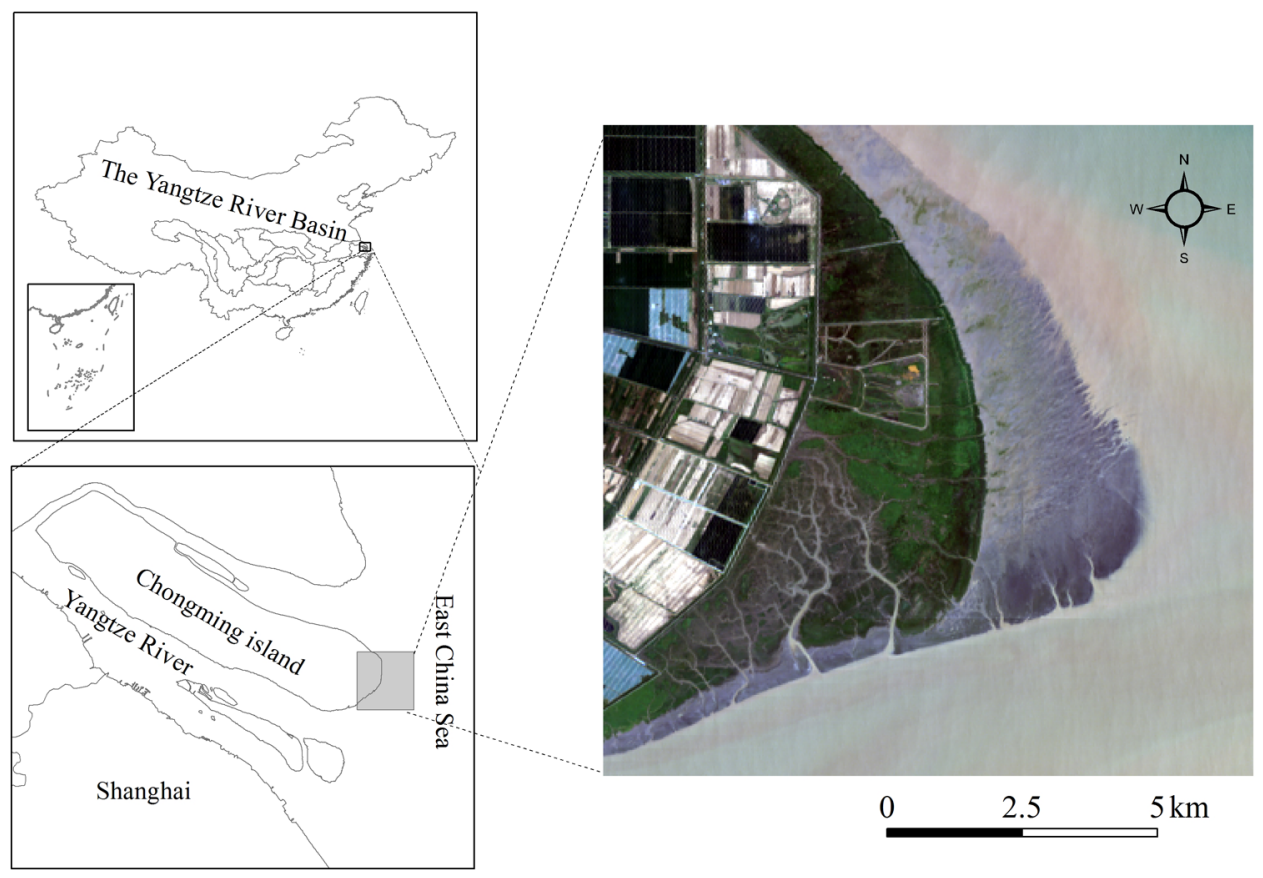

Fig. 1 Location of the study area.

(C) 2016 Society of Photo-Optical Instrumentation Engineers (SPIE) 